\title{
SUBSTELLAR OBJECTS IN NEARBY YOUNG CLUSTERS. VII. THE SUBSTELLAR MASS FUNCTION REVISITED
}

\author{
Alexander Scholz $^{1,2}$, Vincent Geers ${ }^{1}$, Paul Clark ${ }^{3}$, Ray Jayawardhana ${ }^{4,6}$, and KoraljKa Muzic ${ }^{5}$ \\ ${ }^{1}$ School of Cosmic Physics, Dublin Institute for Advanced Studies, 31 Fitzwilliam Place, Dublin 2, Ireland; as110@ st-andrews.ac.uk \\ ${ }^{2}$ School of Physics and Astronomy, University of St Andrews, North Haugh, St Andrews, Fife KY16 9SS, UK \\ ${ }^{3}$ Zentrum für Astronomie der Universität Heidelberg, Institut für Theoretische Astrophysik, Albert-Ueberle-Str. 2, D-69120 Heidelberg, Germany \\ ${ }^{4}$ Department of Astronomy and Astrophysics, University of Toronto, 50 St. George Street, Toronto, ON M5S 3H4, Canada \\ ${ }^{5}$ European Southern Observatory, Alonso de Córdova 3107, Casilla 19, 19001 Santiago, Chile \\ Received 2013 June 5; accepted 2013 August 2; published 2013 September 16
}

\begin{abstract}
The abundance of brown dwarfs (BDs) in young clusters is a diagnostic of star formation theory. Here we revisit the issue of determining the substellar initial mass function (IMF) based on a comparison between NGC 1333 and IC348, two clusters in the Perseus star-forming region. We derive their mass distributions for a range of model isochrones, varying distances, extinction laws, and ages with comprehensive assessments of the uncertainties. We find that the choice of isochrone and other parameters have significant effects on the results, thus we caution against comparing IMFs obtained using different approaches. For NGC 1333, we find that the star/BD ratio $R$ is between 1.9 and 2.4 for all plausible scenarios, consistent with our previous work. For IC348, $R$ is found to be between 2.9 and 4.0, suggesting that previous studies have overestimated this value. Thus the star-forming process generates about 2.5-5 substellar objects per 10 stars. The derived star/BD ratios correspond to a slope of the power-law mass function of $\alpha=0.7-1.0$ for the $0.03-1.0 M_{\odot}$ mass range. The median mass in these clusters-the typical stellar mass-is between 0.13 and $0.30 M_{\odot}$. Assuming that NGC 1333 is at a shorter distance than IC348, we find a significant difference in the cumulative distribution of masses between the two clusters, resulting from an overabundance of very low mass objects in NGC 1333. Gaia astrometry will constrain the cluster distances better and will lead to a more definitive conclusion. Furthermore, the star/BD ratio is somewhat larger in IC348 compared with NGC 1333, although this difference is still within the margins of error. Our results indicate that environments with higher object density may produce a larger fraction of very low mass objects, in line with predictions for $\mathrm{BD}$ formation through gravitational fragmentation of filaments falling into a cluster potential.
\end{abstract}

Key words: brown dwarfs - stars: formation - stars: luminosity function, mass function - stars: pre-main sequence

Online-only material: color figures

\section{INTRODUCTION}

Brown dwarfs (BDs) are an ubiquitous outcome of the star formation process. All young regions investigated so far with sufficient depth host a population of BDs with masses down to $0.01 M_{\odot}$ or even below. The mechanism that governs their formation, however, remains unknown. It is clear that additional physics needs to be included in the models for the cloud fragmentation and subsequent evolution, to allow for the formation of a sizable number of BDs (Bonnell et al. 2007). Plausible options for these processes include fragmentation driven by turbulence, dynamical ejection of embryonic BDs from multiple systems, fragmentation of filaments falling into a cluster potential, or fragmentation of protoplanetary disks, again combined with ejection (Whitworth et al. 2007; Bonnell et al. 2008; Stamatellos \& Whitworth 2009). Young BDs are a critical population to test the relevance of these processes.

The standard diagnostic to distinguish between theoretical scenarios is the distribution of stellar and substellar masses after star formation is finished, or the initial mass function (IMF). In the literature, several parameterizations for the IMF are used, for example, a series of power laws (Kroupa 2001) or a lognormal form (Chabrier 2003). For our goal of determining the abundance of BDs, an often-used parameterization of the IMF is the star/BD ratio $R$, the ratio of the number of objects in the two mass bins from 0.08 to $1.0 M_{\odot}$ and from 0.03 to $0.08 M_{\odot}$, where

\footnotetext{
6 Principal Investigator of SONYC.
}

the low mass cutoff of $0.03 M_{\odot}$ is chosen to assure completeness. The upper mass limit for the stars of $1.0 M_{\odot}$ is to some extent an arbitrary definition, but the relatively small number of highermass stars in the nearby star-forming region assures that this particular choice does not affect the result much. The star/BD ratio as a metric has the advantage of maximizing the sample size in the substellar regime and thus minimizing the statistical errors. Because the stellar side of the IMF is well-determined for the nearby star-forming regions and shows, in the overwhelming majority of regions, no evidence for environmental differences (Bastian et al. 2010), any variation in the star/BD ratio from one region to another would indicate a change in the $\mathrm{BD}$ abundance.

Measuring the substellar mass function and the star/BD ratio is a challenging task. It needs a consistent survey procedure and a careful analysis of possible incompleteness, but the core problem is to estimate the masses. This requires one to make assumptions about the distance to and age of the region, as well as the extinction law used to deredden the photometry or spectra. Furthermore, the conversion from observed quantities to masses can only be done in the framework of a given theoretical isochrone and thus depends on the status of the evolutionary models for young stars and BDs.

In previous work within the project SONYC ("Substellar Objects in Nearby Young Clusters"), we have presented tentative evidence for regional differences in the star/BD ratio. In particular, for the young cluster NGC 1333 in the Perseus starforming complex we find $R \sim 2-3$ based on a very deep survey with comprehensive spectroscopic follow-up (Scholz 
et al. 2009, hereafter SONYC-I; Scholz et al. 2012, hereafter SONYC-IV). For other regions, we and other groups have published $R$-values ranging from 2 to 8 (see SONYC-IV). One of the most extreme cases in the literature is the cluster IC348, the second embedded cluster in Perseus and a slightly older sibling to NGC 1333 (Bally et al. 2008), with a star/BD ratio of $R \sim 8$ (Luhman et al. 2003; Andersen et al. 2008). At face value, this indicates changes in the BD abundance by a factor of four occurring within the same star-forming association. So far, however, the uncertainties for the star/BD ratio have not been assessed accurately. The goal of this paper is to carry out a benchmark test for the two extreme cases NGC 1333 and IC348 to verify their discrepant star/BD ratios.

\section{THE APPROACH}

The core idea of this paper is to determine the mass distribution and the star/BD ratio for two young clusters in Perseus and to assess the associated uncertainties. We will start with consistently selected samples for the two clusters, i.e., samples that have been put together in a homogeneous way, to minimize the influence of selection biases and incompleteness. For these samples, we will then define a consistent way of estimating object masses by comparing photometry with a given model isochrone. In addition to the choice of the isochrone, the distance to the cluster, the extinction law, and the age of the region enter as free parameters into this procedure. We will define a set of scenarios with plausible choices for these parameters and different isochrones. We will then estimate object masses and calculate the star/BD ratio and other indicators of the mass distribution for each of these scenarios. This will yield a useful dataset to discuss the uncertainties in these indicators and assess whether there is evidence for regional differences in the substellar IMF between NGC 1333 and IC348.

\subsection{The Samples}

We use the Spitzer-selected sample of young stellar/substellar objects presented by Gutermuth et al. (2009) which includes our two target regions. For each of the regions, a region of $25^{\prime} \times 25^{\prime}$ centered on the core of the cluster was observed. The selection in Gutermuth et al. (2009) is based on colors and magnitudes in Spitzer and Two Micron All Sky Survey (2MASS) bands from 1 to $24 \mu \mathrm{m}$. Their multi-color selection process uses a series of criteria designed to exclude background stars, nonstellar emission features and extragalactic objects. According to their analysis, this process yields only minimal contamination (a few percent). An earlier version of this process was used in Gutermuth et al. (2008) in a survey of NGC 1333. Assuming that the distances to the clusters are similar, the identical selection procedure also ensures that the depth and completeness in terms of magnitudes in the two samples is comparable. This removes a major obstacle for an accurate comparison of the BD abundance.

Since the primary selection criterion is excess emission in the infrared, this sample only contains objects with emission from circumstellar material, i.e., either disks or envelopes. It does not include disk less young stars and BDs (Class III objects). Therefore, to infer star/BD ratios, we have to make the assumption that the fraction of objects with disks does not change with object mass in the low-mass regime. As recently shown in Dawson et al. (2013), this is a plausible assumption for many star-forming regions, including IC348. For NGC 1333, there might be a slight mass dependence, as the disk fraction in the total Spitzer-selected sample is found to be $83 \% \pm 11 \%$
(Gutermuth et al. 2008), whereas the value for the very low mass objects is only $55 \%-66 \%$ (SONYC-IV). This indicates that the star/BD ratio in this cluster could be slightly overestimated.

The entire Perseus cloud, including the two target clusters have also been observed as part of the Spitzer Legacy program "From Cores to Disks" (C2D, PI: N. Evans). Their Perseus YSO catalog derived from IRAC photometry is discussed in detail in Jørgensen et al. (2006). We prefer to use the Gutermuth et al. (2009) selection, because it is slightly deeper, which is beneficial for our purposes. The downside of the Gutermuth et al. (2009) sample is the limited spatial coverage. Here the C2D catalog is useful to check the spatial completeness of our samples.

In Figure 1 we show the spatial distribution of the selected objects in NGC 1333 and IC348. With red squares we plot the samples from Gutermuth et al. (2009), with black crosses the C2D sample that covers the entire Perseus region. The figure shows that the number of YSO candidates outside the region covered by Gutermuth et al. (2009) is small compared with the total population. This is particularly true for NGC 1333 which shows a very compact profile and can be considered to be spatially complete (see also SONYC-IV). For IC348, there is a tail of a YSO population toward the south-west, which represents the transition region to another densely populated area in the Perseus star-forming region (Cambrésy et al. 2006). In addition, there are about 10 objects outside the coverage of the Gutermuth et al. (2009) survey. However, there is also a large number of additional YSO candidates only contained in the C2D sample within the cluster core. We checked the objects only in C2D and found that they do not show an obvious magnitude or extinction bias with respect to the sample we are using, thus, even in case we are missing members in the outskirts of the cluster, this is not going to affect our analysis in any significant way. While Muench et al. (2003) do find a difference in the IMF between the core and the halo in IC348, both regions (in their definitions) are within the survey area of Gutermuth et al. (2009). We conclude that the samples we are using are not affected by a spatial bias.

Figure 1 also illustrates one major difference between IC348 and NGC 1333. The core of IC348 has about twice the diameter of the core of NGC 1333 (2.1 versus 1.2 pc, Gutermuth et al. 2009), i.e., the cluster volume in IC348 is about 8 times larger than in NGC 1333. On the other hand, IC348 has only about 20\% more YSOs than NGC 1333, according to the Spitzer surveys (Jørgensen et al. 2008; Gutermuth et al. 2009). The fraction of diskless Class III objects is higher in IC348, taken into account that the total the YSO population in IC348 could be up to twice as large as in NGC 1333. This still implies that the object density in NGC 1333 is 4-7 times higher than in NGC 1333. Given the age, size, and number of members in these clusters, this difference is likely to be primordial and not caused by dynamical evolution (see Figure 1 in Gieles et al. 2012). Hence, these two clusters constitute an excellent test case to probe the effects of dynamical interactions and cluster potential on the formation of BDs.

\subsection{Estimating Masses}

For the overwhelming majority of young objects, masses can only be estimated indirectly by comparing an observed quantity with predictions from theoretical isochrones for a given age. For the observed quantity, there are two options, either the effective temperature or the luminosity (or a photometric proxy). The luminosity has the problem that model derivations are sensitive to the age for pre-main sequence objects that are still contracting. In addition, measurements can be affected by extinction as well 

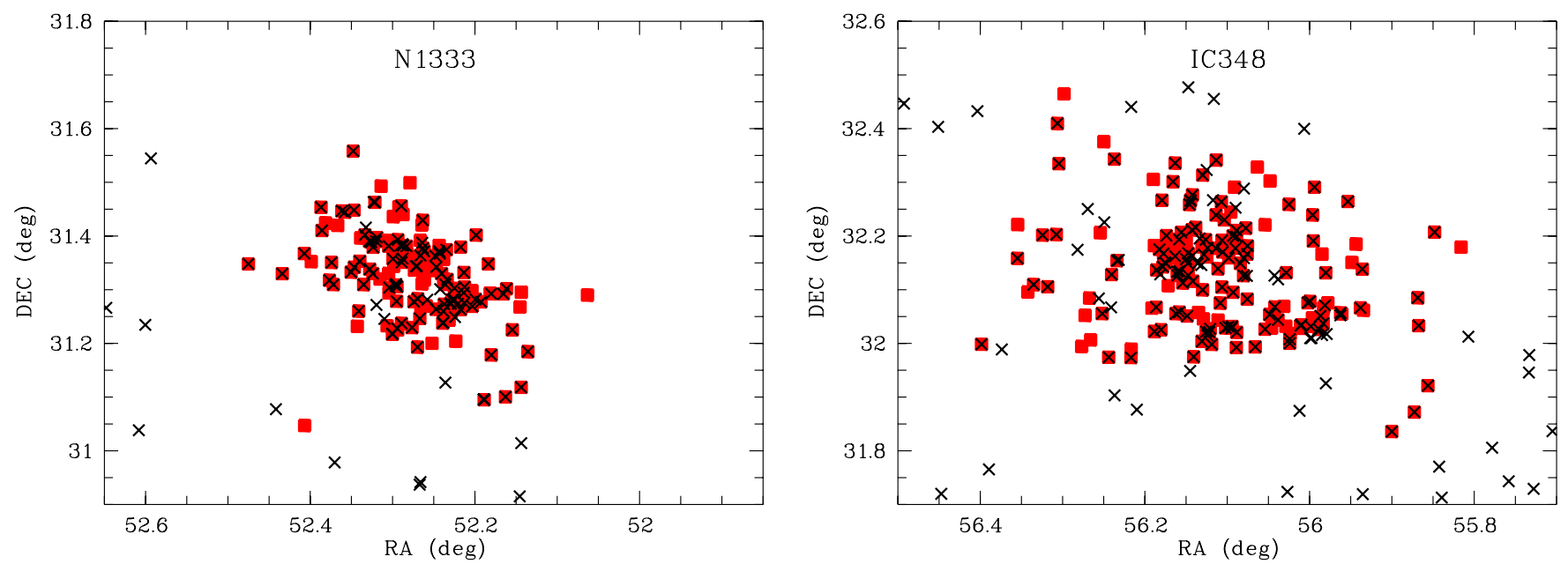

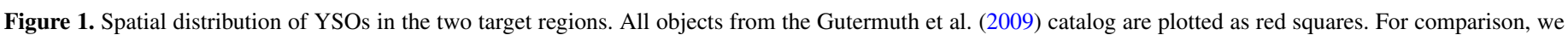
also show the YSO candidates from Jørgensen et al. (2006) as black crosses. Note that the scale in the two panels is identical.

(A color version of this figure is available in the online journal.)

as excess emission from disk and/or accretion. The effective temperature is problematic for other reasons; it depends on atmosphere models and can be altered by magnetic activity (Stassun et al. 2012). This can lead us to underestimate object masses by up to a factor of two.

For our chosen samples, accurate multi-band photometry is available, while the spectroscopic follow-up is not complete. Therefore, we will rely on photometry in the optical and near-infrared to estimate masses. We complement the 2MASS photometry provided by Gutermuth et al. (2009) with optical photometry from Luhman (1999) and Luhman et al. (2003) for IC348 (Landolt $R$ and $I$ band) and from SONYC-I for NGC 1333 (Sloan $i$ and $z$ band). ${ }^{7}$ For some objects without 2MASS nearinfrared magnitudes, we were able to complement the dataset using the photometry from Muench et al. (2003) for IC $348^{8}$ and SONYC-I for NGC 1333. In total, the samples contain 142 (for IC348) and 95 (for NGC 1333) objects with photometry in $J H K$, with smaller subsets of 86 (IC348) and 23 (NGC 1333) with additional optical magnitudes available.

For the objects with 2MASS photometry, we also obtain the errors as listed in the database (mostly between 0.03 and $0.05 \mathrm{mag}$ ). Similarly, the photometry from Muench et al. (2003) provides errors for all measurements. For the remaining photometry, errors for individual objects are not reported in the literature. For the optical magnitudes in IC348, we adopt a generic and conservative uncertainty of $0.1 \mathrm{mag}$. For the SONYC-I magnitudes in NGC 1333, we adopt errors of $0.1 \mathrm{mag}$ for the optical bands and 0.05 mag for the near-infrared bands.

Based on the information given in the papers listed above, the error values adopted for the optical photometry should be typical for the samples. However, some objects might be affected by additional uncertainties introduced to calibration imperfections. Since the $z$ and $I$ bands are located at the longwavelength edge of the sensitivity of the optical CCDs, they are highly susceptible to color terms in the calibration, which are difficult to measure with the usual photometric standard stars. This can introduce errors larger than $0.1 \mathrm{mag}$ in individual sources, which cannot be quantified accurately. This issue is a particular problem for very red sources, since most of their

\footnotetext{
7 Available from http://browndwarfs.org/sonyc.

8 Downloaded from http://flamingos.astro.ufl.edu/sfsurvey/datarelease.html.
}

optical flux is emitted in the part of the spectrum where the CCD sensitivity declines.

We derive masses using three different sets of isochrones from the Lyon group, BT-Settl (with AGSS2009 opacities), BT-Dusty (with AGSS2009 opacities), and BT-Nextgen (with GNC93 opacities). ${ }^{9}$ The latter two are updated versions from the standard AMES-Dusty and Nextgen models. The main difference among the three sets is the treatment of dust. In contrast to Nextgen, Dusty includes dust opacities. Settl includes a full dust cloud model. For more information on the isochrones, see Allard et al. (2011, 2001). Note that atmospheric dust becomes a major source of opacity for $T_{\text {eff }} \lesssim 2500 \mathrm{~K}$ (Helling et al. 2008), corresponding to $M \lesssim 0.02 M_{\odot}$ for young BDs, which is the low-mass limit in our analysis, thus the treatment of dust should not have a major effect on our results. The isochrones predict absolute magnitudes as a function of object mass in all photometric bands for which observations are available. They cover the range from $0.02 M_{\odot}$ or below to $1.4 M_{\odot}$ and are available for ages starting from $1 \mathrm{Myr}$, which is adequate for our purposes.

To compare the observed with the predicted magnitudes, we first shift the isochrones from absolute magnitude to the distance of our target regions, which enters here as a free parameter (see Section 2.3). We also re-bin the isochrones to a uniform stepsize of $0.01 M_{\odot}$, using a linear interpolation over a small portion of the isochrone. We then calculate a series of reddened isochrones for $A_{V}=1-20 \mathrm{mag}$ in steps of $1 \mathrm{mag}$. For this step, the choice of the extinction law is important (see Section 2.3). The upper limit is chosen to be $20 \mathrm{mag}$, for two reasons. First, independent studies indicate that only a small fraction in the Perseus star-forming complex exceeds this extinction value (Lombardi et al. 2010). Second, beyond this value the samples are biased toward bright sources, thus, incompleteness becomes an issue.

After these preparations, the best fit for mass and $A_{V}$ is determined with a $\chi^{2}$ minimization. The number of degrees of freedom in this process is the number of photometric bands for which data is available $(N=3-5)$ minus the number of free parameters (mass and $A_{V}$, i.e., 2). For each object, we saved the combination of mass and $A_{V}$ that results in the minimum

\footnotetext{
9 Downloaded from http://phoenix.ens-lyon.fr/simulator/index.faces.
} 

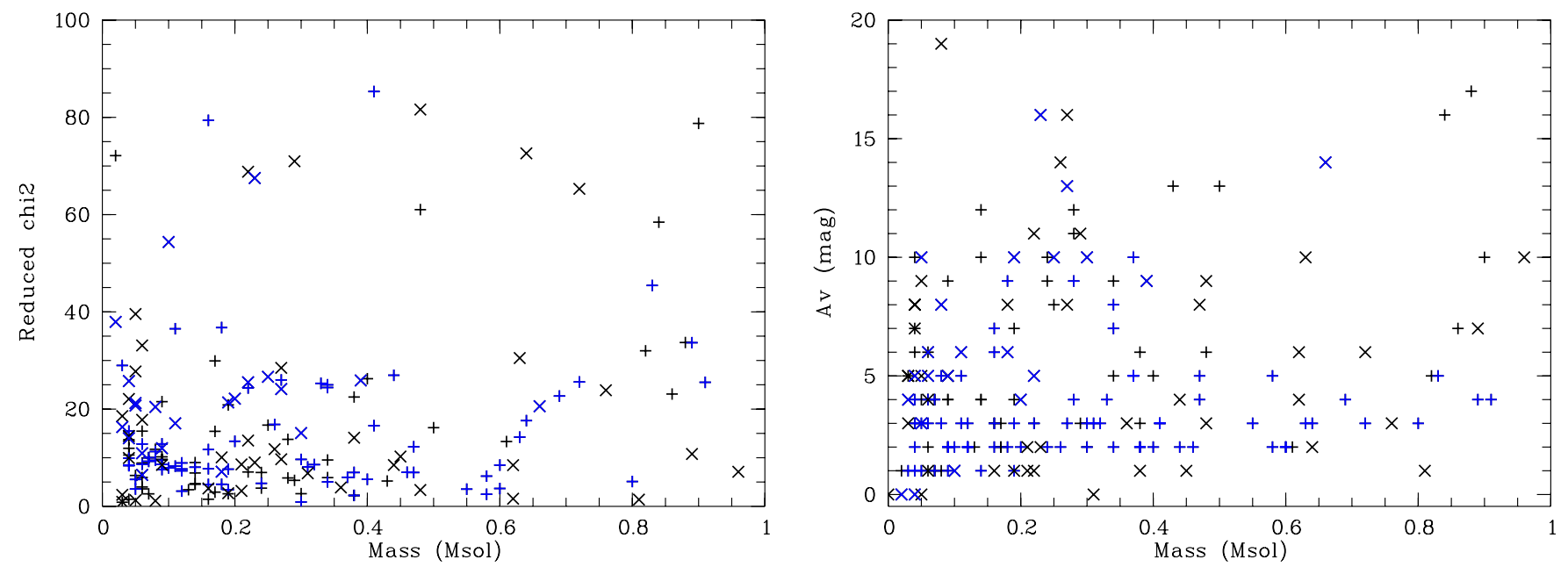

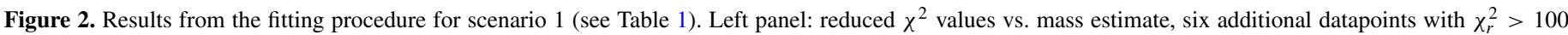

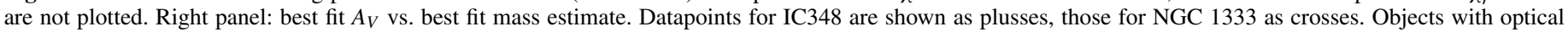
photometry are shown in blue.

(A color version of this figure is available in the online journal.)

value for $\chi^{2}$, the corresponding reduced $\chi^{2}\left(\chi_{r}^{2}\right.$, i.e., $\chi^{2}$ divided by the number of degrees of freedom) and the number of available bands $N$. Objects with best fit value of $A_{V}=20$ are discarded from the analysis-since this is the upper limit in our grid of isochrones, their mass estimate is not reliable. A typical example of the resulting $\chi_{r}^{2}$ plotted versus the best mass estimate is shown in Figure 2, left panel. This figure reveals that the procedure produces similar fitting results for high- and low-mass objects and for objects with and without optical photometry. We also show a typical $A_{V}$ versus mass plot from this procedure (Figure 2, right panel). The upper limit in $A_{V}$ is not changing significantly with mass, i.e., there is no evidence for an extinction bias in these samples (apart from the $A_{V}<20$ cutoff).

The distribution of reduced $\chi^{2}$ can in principle be used to assess the goodness-of fit for our mass estimates. For a good fit, we expect $\chi_{r}^{2}$ to have an average of 1.0 and a standard deviation of $\sim \sqrt{2 / N}$. However, as shown in Figure 2, our procedure yields significantly higher values in $\chi_{r}^{2}$. This indicates that either the model does not reflect the data well or that the errors are underestimated. In our case, the high values for $\chi_{r}^{2}$ are mostly explained by the fact that our model is discretely sampled in mass- $A_{V}$ space. The stepsize in mass and $A_{V}$ results in magnitude steps that are often larger than the typical photometric error. In addition, in some cases the errors of the optical photometry may be underestimated, see above. Therefore, we only use the procedure to select the best fit solution.

\subsection{The Scenarios}

In our estimation of masses from photometry, the distance, age, as well as the extinction law, expressed in the quantity $R_{V}=A_{V} / E_{(B-V)}$, are considered free parameters. In addition, we have to choose the theoretical isochrone. What we call "scenarios" in the following are combinations of isochrone, distance, age, and extinction law for which we estimate masses. These scenarios have been chosen to cover the plausible range of these parameters and to give insight into the impact of the specific choice of a parameter or an isochrone on the mass estimates. In the following, we justify the choice of the range of the parameters.

For the extinction, we use the parameterized law by Cardelli et al. (1989), with $R_{V}=3.1$, the canonical value used for the interstellar matter (ISM). This law yields extinction offsets that are consistent with the often-used extinction values published by Schlegel et al. (1998). In reality, $R_{V}$ depends on the grain properties and is not the same for every line of sight; Cardelli et al. (1989) report values ranging from 2.6 to 5.6, with the overwhelming majority (22 out of 27 cases) below 4.5. We therefore use $R_{V}=4.5$ as an alternative value to be able to assess the impact of the choice of $R_{V}$ on the mass estimates.

The distances to the two clusters are not well constrained. The entire Perseus cloud is usually assumed to have an average distance of $\sim 300 \mathrm{pc}$, which we use as a default value. Based on the Hipparcos parallaxes for the early-type stars, de Zeeuw et al. (1999) estimate $318 \pm 27$ pc for the cloud. Based on a kinematical analysis of a much larger sample of A stars, Belikov et al. (2002) infer 300 pc (270-330 pc). However, there are indications that NGC 1333 is located at a shorter distance. Hirota et al. (2011) report a distance of 235 pc for NGC 1333 based on interferometry of the maser emission from a source that may be associated with the cluster. This is also consistent with an earlier photometric estimate of the distance of NGC 1333 (220 pc) by Cernis (1990). In addition, Lombardi et al. (2010) suggest, based on extinction map analysis, that the northern part of the Perseus region (where IC348 is located) is slightly more distant than the southern part (where NGC 1333 is located). To take this into account, we use a distance of $230 \mathrm{pc}$ as an alternative value, noting that this is only a viable option for NGC 1333.

The ages that are typically quoted are 2-4 Myr for IC348 and 1-3 Myr for NGC 1333 (see Bally et al. 2008 and references therein). Judging from model-independent indicators of evolutionary state (fraction of objects with disks, fraction of objects in Class I stage, luminosity function), NGC 1333 is definitely younger than IC 348, and both are clearly younger than star-forming regions with established ages of 5-10 Myr like Upper Scorpius and the TW Hydrae Association (e.g., Lada et al. 1996; Haisch et al. 2001; Gutermuth et al. 2008). In the context of our study, the relevant quantity is not the age of 
Table 1

Results from Mass Estimates for IC348 and NGC 1333

\begin{tabular}{|c|c|c|c|c|c|c|c|c|c|c|}
\hline Cluster & No & $\begin{array}{c}D \\
(\mathrm{pc})\end{array}$ & $\begin{array}{c}t \\
(\mathrm{Myr})\end{array}$ & Model & $R_{V}$ & $R$ & $\operatorname{Min}_{-} \operatorname{Max}^{\mathrm{a}}$ & Min-Max ${ }^{b}$ & $\alpha$ & $\overline{\bar{M}}$ \\
\hline IC348 & 1 & 300 & 3 & BT-Settl & 3.1 & $3.6(96 / 27)$ & $2.8-4.4$ & $2.6-4.1$ & 0.72 & 0.27 \\
\hline IC348 & 2 & 300 & 1 & BT-Settl & 3.1 & $2.1(88 / 42)$ & $1.7-2.5$ & $1.5-2.9$ & 0.95 & 0.13 \\
\hline IC348 & 3 & 230 & 3 & BT-Settl & 3.1 & $1.9(84 / 45)$ & $1.5-2.2$ & $1.3-2.1$ & 1.00 & 0.16 \\
\hline IC348 & 4 & 300 & 3 & BT-Settl & 4.5 & $3.2(94 / 29)$ & $2.6-4.0$ & $2.7-4.3$ & 0.76 & 0.26 \\
\hline IC348 & 5 & 300 & 3 & BT-Nextgen & 3.1 & $4.0(99 / 25)$ & $3.1-4.9$ & $3.4-5.5$ & 0.67 & 0.19 \\
\hline IC348 & 6 & 300 & 3 & BT-Dusty & 3.1 & $2.9(92 / 32)$ & $2.3-3.5$ & $2.4-3.4$ & 0.81 & 0.22 \\
\hline N1333 & 1 & 300 & 3 & BT-Settl & 3.1 & $2.2(43 / 20)$ & $1.6-2.8$ & $1.9-2.5$ & 0.94 & 0.27 \\
\hline N1333 & 2 & 300 & 1 & BT-Settl & 3.1 & $2.1(47 / 22)$ & $1.6-2.7$ & $2.0-2.5$ & 0.94 & 0.13 \\
\hline N1333 & 3 & 230 & 3 & BT-Settl & 3.1 & $2.4(47 / 20)$ & $1.8-3.0$ & $2.2-2.4$ & 0.90 & 0.18 \\
\hline N1333 & 4 & 300 & 3 & BT-Settl & 4.5 & $2.0(43 / 21)$ & $1.6-2.6$ & $1.9-2.5$ & 0.96 & 0.30 \\
\hline N1333 & 5 & 300 & 3 & BT-Nextgen & 3.1 & $2.2(44 / 20)$ & $1.7-2.8$ & $1.9-2.6$ & 0.93 & 0.18 \\
\hline N1333 & 6 & 300 & 3 & BT-Dusty & 3.1 & $1.9(42 / 22)$ & $1.5-2.5$ & $1.8-2.0$ & 0.99 & 0.22 \\
\hline
\end{tabular}

Notes.

a Statistical uncertainties for $R$, only dependent on sample size.

${ }^{\mathrm{b}}$ Min-max range of $R$ based on the number of objects close to the substellar limit, see Section 2.4.

the cluster, but the average age of the objects contained in our samples, which may not include the youngest, embedded population because we require a near-infrared detection. In fact, we showed in SONYC-IV that most of the very low mass objects in NGC 1333 are consistent with an age of 1-5 Myr, based on their position in the Hertzsprung-Russell diagram (HRD). Therefore, we use a default value of $3 \mathrm{Myr}$, which is plausible for both clusters. To assess the influence of the age on the mass estimates, we additionally estimate masses for an age of $1 \mathrm{Myr}$.

To evaluate the impact of the choice of the parameters, we define six scenarios for which we estimate object masses. These scenarios are listed in Table 1 . The default scenario 1 uses a distance of $300 \mathrm{pc}$, an age of $3 \mathrm{Myr}, R_{V}$ of 3.1 , and the BT-Settl model, which has the most recent opacities and the most advanced treatment of dust. In scenarios 2-4 we vary the cluster parameters. In scenario 2, we use the younger age of $1 \mathrm{Myr}$; in scenario 3, the alternative distance of $230 \mathrm{pc}$; and in scenario 4 , the alternative value for $R_{V}$ of 4.5 . In scenarios 5 and 6 , we switch to the Nextgen and DUSTY isochrones.

From the resulting mass distribution in a given scenario, we derive the cumulative distribution of object masses, i.e., the fraction of objects below a given mass, as a function of mass. These plots are shown in Figure 3. For each scenario, we determine the number of objects with $0.03 \leqslant M \leqslant 0.08 M_{\odot}$ (BDs) and with $0.08<M<1.0 M_{\odot}$ (stars) and calculate the star/BD ratio $R$. To be able to compare with the literature, we also calculate the slope of the mass function $\alpha$ for the power-law parameterization $d N / d M \propto M^{-\alpha}$, directly from the star/BD ratios, i.e., using two bins in mass, one for BDs and one for stars. In addition, we derive the median mass $\bar{M}$ for each scenario. The resulting parameters for the six scenarios are given in Table 1.

\subsection{Error Budget}

An important part of this work is to evaluate the errors in the derived parameters. In our chosen experiment, five factors contribute to the uncertainties.

1. Sample size. The part that is easiest to quantify is the statistical uncertainty, which is purely determined by the sample size. For this paper, we use the same approach as in SONYC-IV, which is based on the IDL scripts presented in Cameron (2011). In short, we calculate Bayesian confidence intervals from the beta distribution. We note that for large samples this procedure gives results that are very similar to binomial confidence intervals. The resulting values are listed in Table 1, Column 8. Typically, the sample size introduces an error in $R$ of about $\pm 0.3-0.9$ for IC 348 and $\pm 0.5-0.6$ for NGC 1333 .

2. Models. Masses are only defined in relation to evolutionary models, and at young ages the deficiencies of the available tracks are well documented (Baraffe et al. 2002; Wuchterl $\&$ Tscharnuter 2003). However, at the moment no tracks with self-consistent treatment of the collapse and infall are available to the community. ${ }^{10}$ Based on a dynamical mass estimate for the very low mass pre-main sequence object $\mathrm{AB}$ Dor $\mathrm{C}$, which is older than our target regions, Close et al. (2005) claim that the existing mass-luminosity relations underestimate masses by a factor of about two for young objects, but this claim has been questioned (Luhman $\&$ Potter 2006). At young ages and very low masses, the only direct benchmark test for these tracks is the eclipsing BD binary 2M J05352184-0546085 (Stassun et al. 2007). The Baraffe et al. isochrones fail to reproduce the surprising temperature reversal in this object (i.e., the more massive object is cooler than the secondary), an effect that is likely related to the presence of strong magnetic fields on the primary (Stassun et al. 2012). The luminosities of the two components, however, are consistent with the isochrones. Irwin et al. (2007) have discovered and analyzed a very young eclipsing binary with component masses around $\sim 0.2 M_{\odot}$; that system confirms the isochrones within the errorbars as well. Thus, although more work is required to calibrate the isochrones, some preliminary trust in their validity seems warranted.

3. Cluster parameters. As discussed in Section 2.2, several properties of the clusters affect the mass estimates, in particular the distance, the age, and the extinction law. From our set of scenarios documented in Table 1 (scenarios 1-4) we can assess how the uncertainties in these parameters propagate through the procedure. In general, changes in

\footnotetext{
10 If, in a hypothetical future, evolutionary tracks are linked with the initial conditions via realistic models for collapse, infall, and dynamical evolution in clusters, one would directly compare the predicted with the observed luminosity functions or HRDs. The semi-empirical estimate of IMFs for star-forming regions, as it is done in this paper and in many others in the literature, would become obsolete.
} 

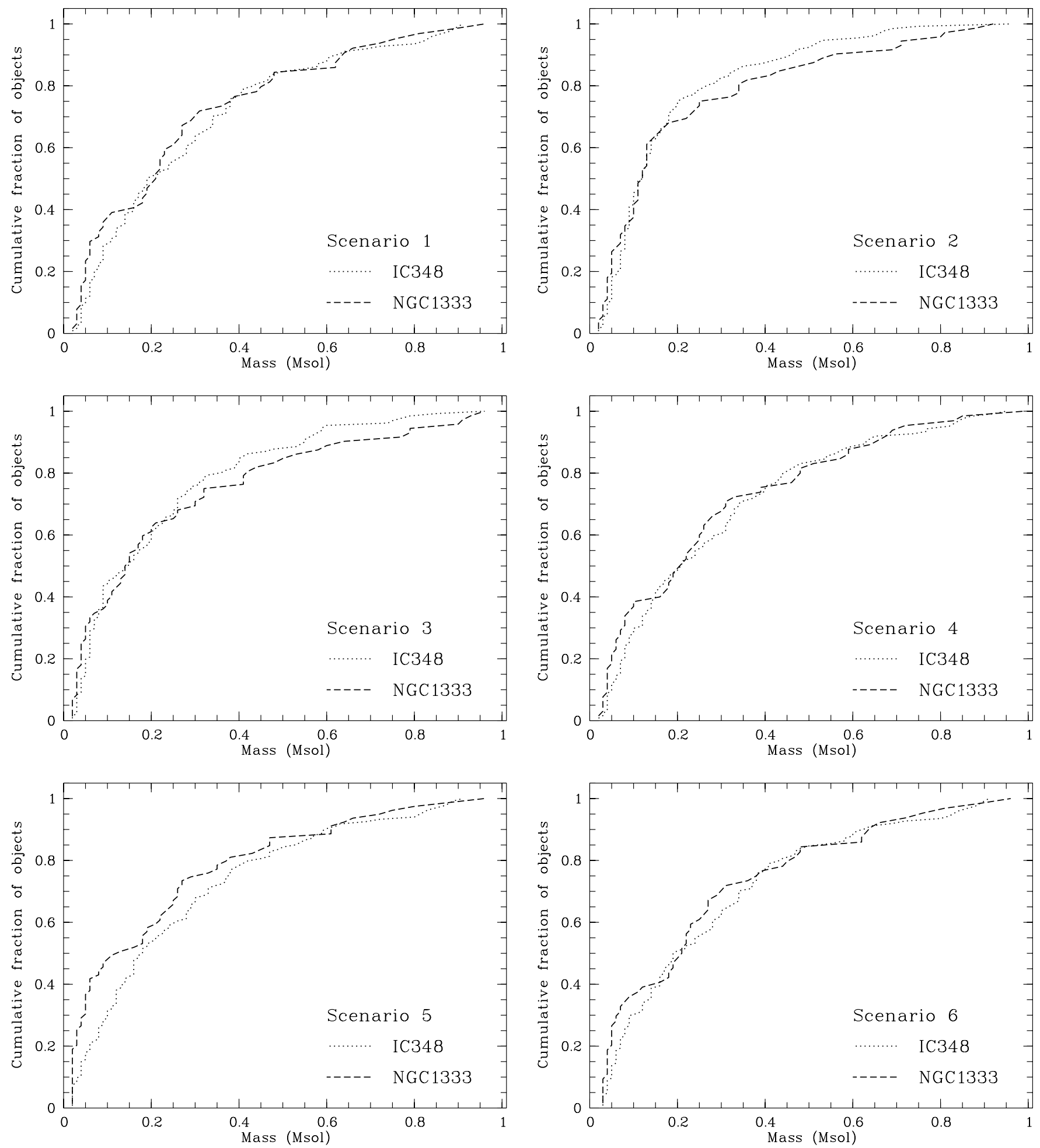

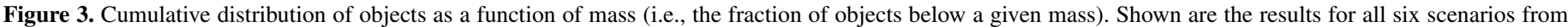
Table 1 . The parent sample for these plots has $A_{V}<20$ and $M \leqslant 1.0 M_{\odot}$.

age and distance cause significant changes in the estimated mass distribution, while a change in the extinction does not. For NGC 1333, the induced variations in the star/BD ratios are small; $R$ varies from 2.0 to 2.4 , smaller than the statistical uncertainties. For IC348, the scatter is larger, from 1.9 to 3.6 , but excluding the implausible scenarios with age of $1 \mathrm{Myr}$ and distance of $230 \mathrm{pc}$ this range shrinks to 3.2-3.6. We note that the star/BD ratio increases somewhat with assumed age. For example, for IC348 and an (unlikely) age of $5 \mathrm{Myr}$ we obtain $R=6.7$. This is easy to understand-as the objects evolve, they become fainter, i.e., the same magnitude corresponds to a larger mass. As a result, objects move from the substellar to the stellar domain, and the star/BD ratio increases.

4. Completeness. The samples we are using have the same depth and completeness, in terms of magnitudes, which 
Table 2

Probability that Two Cumulative Distributions of Object Masses are Drawn from the Same Parent Distribution

\begin{tabular}{lcccccc}
\hline \hline Scenario & $\begin{array}{c}\text { N1333-1 } \\
\text { Default }\end{array}$ & $\begin{array}{c}\text { N1333-2 } \\
t=1 \mathrm{Myr}\end{array}$ & $\begin{array}{c}\text { N1333-3 } \\
D=230 \mathrm{pc}\end{array}$ & $\begin{array}{c}\text { N1333-4 } \\
R_{V}=4.5\end{array}$ & $\begin{array}{c}\text { N1333-5 } \\
\text { Nextgen }\end{array}$ & $\begin{array}{c}\text { N1333-6 } \\
\text { Dusty }\end{array}$ \\
\hline IC348-1: default & 0.26 & $\mathbf{0 . 0 2}$ & $\mathbf{0 . 0 4}$ & 0.50 & $\mathbf{0 . 0 0 1}$ & 0.17 \\
IC348-2: $t=1 \mathrm{Myr}$ & $\mathbf{0 . 0 0 2}$ & 0.49 & 0.10 & $\mathbf{0 . 0 0 3}$ & $\mathbf{0 . 0 1}$ & $\mathbf{0 . 0 0 2}$ \\
IC348-3: $D=230 \mathrm{pc}$ & 0.32 & 0.33 & 0.32 & 0.39 & $\mathbf{0 . 0 3}$ & 0.32 \\
IC348-4: $R_{V}=4.5$ & 0.26 & $\mathbf{0 . 0 0 3}$ & 0.05 & 0.13 & $\mathbf{0 . 0 0 1}$ & 0.17 \\
IC348-5: Nextgen & 0.80 & $\mathbf{0 . 0 1 6}$ & 0.30 & 0.76 & $\mathbf{0 . 0 2}$ & 0.54 \\
IC348-6: Dusty & 0.47 & $\mathbf{0 . 0 2}$ & 0.08 & 0.74 & $\mathbf{0 . 0 0 4}$ & 0.32 \\
\hline
\end{tabular}

Note. Combinations with significant differences are marked.

eliminates one major source of uncertainty. However, in terms of object masses the depth of the survey depends on the assumed distance and age. Assuming a shorter distance, as well as a younger age, will produce lower masses for the same magnitudes, i.e., the entire mass distribution would be shifted to lower masses, including the limits for completeness. For our default scenario with age of $3 \mathrm{Myr}$ and distance of $300 \mathrm{pc}$, the magnitude limit of the samples corresponds to $\sim 0.02 M_{\odot}$. The alternative distance of $230 \mathrm{pc}$ (scenario 3 ) implies a magnitude shift by $0.6 \mathrm{mag}$. According to BT-Settl isochrones, this translates to a mass limit of $0.015 M_{\odot}$. The alternative age of $1 \mathrm{Myr}$ (scenario 2) yields a new mass limit of $0.012-0.015 M_{\odot}$. Thus, in these scenarios we would be sensitive to slightly lower mass objects, but since the object density is very low in this mass domain, this has only a minuscule effect on the mass distribution. Furthermore, it is not going to affect the star/BD ratios.

5. Degeneracy. In many cases there are multiple mass $/ A_{V}$ combinations that fit the data with a similar $\chi^{2}$. In particular, for an object with a best mass estimate around the hydrogen burning limit $\left(0.07-0.09 M_{\odot}\right)$ this method is unable to distinguish between a star and a BD. As an estimate of the introduced uncertainty in $R$ we selected all objects in this mass regime and included them first in the BD count (for the lower limit of the star/BD ratio) and then in the star count (for the upper limit). The resulting ranges in $R$ are listed in Table 1, Column 9. These intervals are $\pm 0.1-0.3$ for NGC 1333 and $\pm 0.5-1.0$ for IC 348 . We note that the degeneracy is less of a problem for the two other mass thresholds involved in the calculation of $R(0.03$ and $\left.1.0 M_{\odot}\right)$, simply because the number of objects around these limits is low.

Combining these error sources, the values of $R$ are affected by an uncertainty of approximately \pm 1 for the two samples studied here. Currently this is about the best that can be done in terms of estimating this indicator for young star-forming regions. This translates to an uncertainty of about \pm 0.1 in the power-law slope $\alpha$. The median of the mass function can be estimated with an accuracy of $\pm 0.1 M_{\odot}$ for nearby star-forming regions.

Looking ahead, there are obvious ways to lower these uncertainties. First, future evolutionary tracks need to include realistic initial conditions and require more detailed calibration (e.g., with eclipsing binaries). Second, the extinction parameters need to be studied in more detail for individual regions. Third, independent estimates for the (relative or absolute) ages of young clusters are needed. Fourth, the accuracy in the distances of these clusters (and many other well-studied star-forming regions) should be improved. In this respect, the Gaia satellite will be a major opportunity, as it is anticipated to be able to measure distances with $1 \%$ accuracy for open clusters within $1 \mathrm{kpc}$ (Prusti 2011), a huge improvement over the current estimates. Fifth, it is a worthwhile goal to obtain comprehensive sets of multi-filter photometry for star-forming regions. And sixth, additional survey work in rich star-forming regions with significantly more members (a factor of 10 or more) than the well-studied nearby regions can be used to minimize the statistical uncertainties. However, since these regions are only found at large distances of $>2 \mathrm{kpc}$, such studies have to be postponed until larger facilities, namely James Webb Space Telescope or Extremely Large Telescopes (ELTs), are available.

\section{RESULTS}

\subsection{The Cumulative Distribution}

Our procedure yields for each scenario and for each cluster a distribution of masses. Prior to calculating parameters for the IMF, we examine these distributions directly. In Figure 3, we show the cumulative distribution of object masses, i.e., the fraction of objects below a given mass, as a function of mass for all six scenarios. We compare the 36 combinations of the functions shown in Figure 3 (six for IC348 and six for NGC 1333) with a Kolmogorov-Smirnov test to search for differences in the mass distribution. By doing that, we test the null hypothesis that two given distributions are drawn from the same parent distribution. In Table 2, we list the probabilities that this hypothesis is valid.

For 14 out of 36 combinations, this probability is $<5 \%$, i.e., the null hypothesis should be rejected. Six of them are combinations that include scenario 5 for NGC 1333, i.e., the one that uses the Nextgen isochrone. This scenario produces an unusual large number of low-mass BDs $\left(0.02-0.05 M_{\odot}\right)$ for NGC 1333, which causes the discrepancy with other distributions. This shows that apparent differences in the mass distribution of young clusters can be introduced simply by the choice of the isochrone. We caution against comparing mass distributions derived with inconsistent isochrones.

Seven further combinations of scenarios with significant differences in the mass distribution include scenario 2 for one of the clusters, i.e., the scenario with an age of 1 Myr. As an example, we show in Figure 4 (left panel) a comparison between the default scenario for IC348 and scenario 2 for NGC 1333. From this figure the origin of the difference is clear-the mass distribution for NGC 1333 shows a pronounced "knee" at $0.15 M_{\odot}$. However, the mass distribution in IC348 gives almost exactly the same "knee" when assuming an age of 1 Myr (upper left panel in Figure 3). This effect is best explained by the "knee" in the mass-luminosity relation at this age, which is 

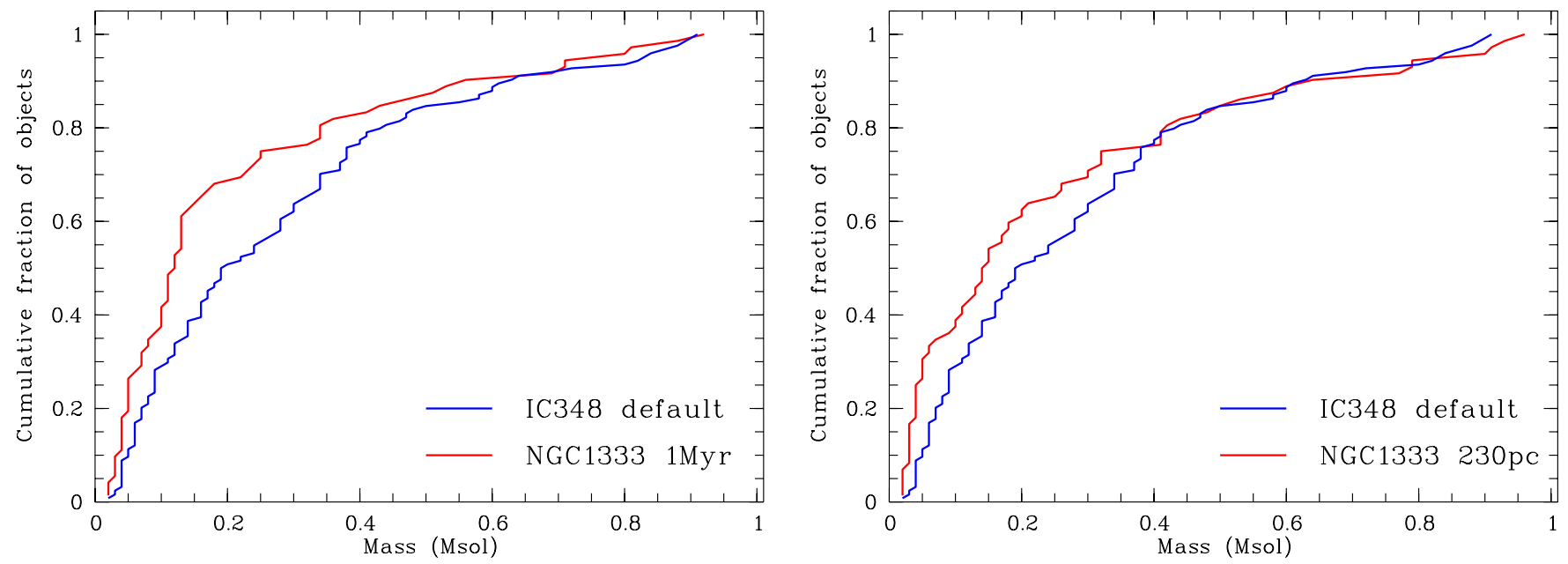

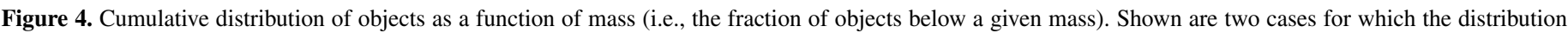

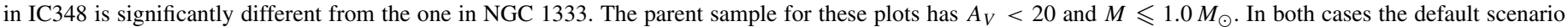

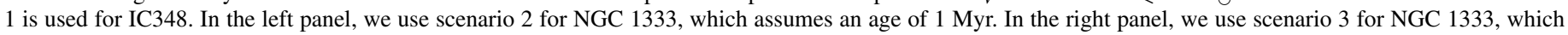
assumes a distance of $230 \mathrm{pc}$.

(A color version of this figure is available in the online journal.)

predicted to become weaker with age. The resulting differences in the mass distribution cannot be attributed to environmental differences.

The combination of the default scenario for IC348 and scenario 3 for NGC 1333 produces a significant difference as well (see Figure 4, right panel). This combination assumes the shorter distance of $230 \mathrm{pc}$ for NGC 1333. We note that three further combinations with this assumption give marginally significant differences with probabilities between $5 \%$ and $10 \%$. As explained in Section 2.3, multiple independent studies indicate that NGC 1333 is located at a shorter distance than IC348, thus, this scenario is plausible.

In summary, his comparison shows that the choice of the cluster parameters and the choice of the isochrone can have noticeable effects on the estimated distribution of object masses. We find that there could be significant differences in the mass distributions between the two clusters, if NGC 1333 is at a shorter distance than IC348. In this case, our analysis indicates a larger proportion of very low mass objects with masses $<0.3 M_{\odot}$ in NGC 1333.

\subsection{The Star/BD Ratio}

From the mass distributions in the six scenarios we calculated the star/BD ratio, see Table 1 for the results. For the cluster NGC 1333, our six scenarios give star/BD ratios of 1.9-2.4, which is a range comparable to the statistical uncertainties (see Section 2.4). This means that our previous estimate for this cluster from SONYC-IV $(R \sim 2.3)$ is confirmed.

For IC348, we find a wider range of values between 1.9 and 4.0. These values imply that star/BD ratios reported in the literature for IC348 of $\sim 8$ (Luhman et al. 2003; Andersen et al. 2008) are overestimated. A possible reason for these large values is survey incompleteness or low number statistics. As explained in Section 2.4, the star/BD ratio depends on age, therefore these large star/BD ratios in the literature would become viable if IC348 is, in fact, significantly older than 3 Myr. This is unlikely, as age-dependent observable quantities like the disk fraction are comparable to other 2-3 Myr old clusters (Dawson et al. 2013).

An age of $1 \mathrm{Myr}$ and a distance of $230 \mathrm{pc}$ are not plausible for IC348 (Section 2.3). Excluding the scenarios using these parameters gives a range for $R$ between 2.9 and 4.0, which is our best estimate for this cluster. These values are somewhat larger than in NGC 1333, although still within the margin of error. For example, for the default case, the star/BD ratio is 3.6 for IC348 with a lower limit of 2.6, and 2.2 for NGC 1333 with an upper limit of 2.5. This is before taking into account the statistical uncertainties. Thus, based on the star/BD ratio the evidence for regional differences in the mass distribution of IC348 and NGC 1333 is tentative.

\subsection{Other Parameters}

In Table 1 we also report two other quantities that are used in the literature to describe the IMF. The power-law slope of the mass function $\alpha$ is directly determined from the star/BD ratio and thus reflects the same trends reported in Section 3.2. For NGC $1333, \alpha$ is $0.9-1.0$; for IC349 $0.7-1.0$, or $0.7-0.8$ after excluding the implausible scenarios. Note that the slope is an average value for the mass range $0.03-1.0 M_{\odot}$; therefore it is not unexpected to find values somewhere between the typical slope of 1.3 in the regime of low-mass stars (Kroupa 2001) and the typical value of $\sim 0.6$ in the very low mass regime (see SONYC-IV).

Independent from the star/BD ratio, we determine the median mass for each scenario. These values vary between 0.13 and $0.30 M_{\odot}$, again indicating that the choice of the cluster parameters and the choice of the isochrone affect the results considerably. For a given scenario, the two clusters have very similar median masses (maximum difference is $0.04 M_{\odot}$ for scenario 4). Increasing age and distance will also increase the median mass. Note that Alves de Oliveira et al. (2013) have recently determined the mass function for IC 348 from a different survey and find a characteristic mass (in the lognormal mass function) of $0.21-0.22 M_{\odot}$ consistent with the values derived here.

\section{IMPLICATIONS FOR BROWN DWARF FORMATION}

In Section 3 we established that differences in the mass distributions of the two clusters are significant, if NGC 1333 is closer than IC348. In addition, there is tentative evidence that the star/BD ratio in IC348 is slightly larger than in NGC 1333. 
Given that our two target regions differ in object density by a factor of 4-7 (Section 2.2), this finding can in principle be used to put constraints on theories for BD formation in which the stellar density is a critical parameter for the yield.

One popular scenario to form BDs is as part of dynamical cluster formation. Here, very low mass objects are removed from their accretion reservoir by dynamical ejections and thus stop their growth; the final mass is set by the competition between accretion and ejection (Bate 2012). In this model the efficiency of BD formation is partly controlled by the likelihood for dynamical encounters which is related to the object density. The most recent radiation-hydrodynamical simulations by Bate (2012, see their Table 1) are comparable to the clusters studied here in terms of initial cloud mass and number of objects produced. The simulations yield star/BD ratios $(>2.6,>4.1)$ and median masses $\left(0.21,0.24 M_{\odot}\right)$ that are consistent with our empirical results. However, the impact of object density is difficult to judge, since the simulations have only been carried out for a very limited set of initial conditions.

Another way to form BDs that has been suggested in the literature is gravitational fragmentation of infalling gas into a stellar cluster (Bonnell et al. 2008). Here the potential well, and thus the object density in the cluster, is a critical parameter for the efficiency of BD formation. The BDs and very low mass objects are expected to be formed preferentially in regions with high stellar density. Qualitatively the predictions from this scenario are confirmed by our analysis: Under plausible assumptions, the denser cluster NGC 1333 has indeed a larger fraction of very low mass stars and BDs (see Section 3.2).

Their Figure 7 shows the BD fraction as a function of object density from their simulations. The two regions investigated in the current paper are both at the low end of the considered densities $\left(1-100 \mathrm{pc}^{-3}\right)$. For these densities, the predicted BD fractions are between $7 \%$ and $13 \%$. In their paper the BD fraction is calculated as the number of BDs divided by the total number of objects. From our mass distributions, this quantity is $\sim 20 \%$ in IC348 and $~ 30 \%$ in NGC 1333, i.e., the predicted values are lower than the observed one. If this formation mechanism plays a role and the predictions are realistic, it could only contribute about one third to half of the BDs in the clusters. Other mechanisms, for example disk fragmentation followed by the ejection of embryonic or proto-BDs (Stamatellos \& Whitworth 2009; Basu \& Vorobyov 2012), could contribute to the final tally of substellar objects in the young clusters.

Judged by their Figure 7, an increase in the object density by one order of magnitudes would result in an increase in the $\mathrm{BD}$ fraction by a factor of about two. This is consistent with the observed difference between IC348 and NGC 1333, although such a difference is, as explained in Section 3.2, still within the uncertainties. Therefore, the scenario remains viable, but cannot be rigorously verified with the current surveys. An important test for the theory would be the measurement of the BD fraction in a cluster that is significantly denser than NGC 1333, such as RCW38 (Wolk et al. 2008) or the Orion Nebula Cluster. So far, the survey results in the ONC give inconsistent answers regarding the frequency of very low mass objects (Andersen et al. 2011; Da Rio et al. 2012).

The aforementioned scenario for BD formation via disk fragmentation could also result in a star/BD ratio that depends on stellar density, if some of the fragmentation processes are driven by stellar encounters (Thies et al. 2010) or disk-disk collisions (Shen et al. 2010). Stellar encounters could also facilitate the ejection of bound BD companions from their host stars (Goodwin \& Whitworth 2007). With these additional mechanisms, disk fragmentation models would again produce more BDs in a region with higher stellar density, which is qualitatively what we find to be the case. However, the expected magnitude of this effect has not been estimated yet.

As pointed out in Section 2.1, the current stellar densities in IC348 and NGC 1333 are probably representative of their primordial densities (Gieles et al. 2012; Moeckel et al. 2012). With constant star formation rate, these should scale with the gas density in the original cloud. Under these assumptions, we can also put limits on scenarios for BD formation through turbulent fragmentation. According to the model presented by Padoan $\&$ Nordlund (2002), a factor of five in density enhancement should amount to a very large increase (about an order of magnitude, see their Figure 1) in the number of BDs. In the gravoturbulent picture (Hennebelle \& Chabrier 2009) the effect seems to be similar. Qualitatively the result is as seen in the Perseus clusters (i.e., the denser cluster produces more BDs), but the magnitude of the effect is much larger than what we derive. However, differences in other cluster parameters, for example in the Mach number, could partially erase the predicted effect. Since their predictions depend heavily on initial conditions, it is doubtful whether empirically derived IMFs can provide a meaningful test for these models.

An important caveat in our analysis is the fact that what we derive is a snapshot of the mass distribution, which may not necessarily represent the IMF. This is particularly relevant because NGC 1333 is at an earlier evolutionary state than IC348 and might become as rich as its sibling at the other side of the Perseus star-forming complex (Lada et al. 1996). In the typical picture of cluster formation, however, lower mass objects form later (e.g., Bate 2012, their Figure 8), thus, if additional formation processes in NGC 1333 have any effect on the mass distribution, they are expected to amplify the observed discrepancy with IC348. For the comparison with the models quoted above, which typically only predict a mass distribution of cores, not an IMF, this issue is not of practical relevance.

\section{SUMMARY}

We present a systematic study of the mass distribution in the two young open clusters IC348 and NGC 1333, with specific emphasis on the substellar regime. These two regions are of specific interest because NGC 1333 has a higher spatial density (by a factor of 4-7). In the following we list our most important findings.

1. The mass distribution as well as the parameters derived from it, e.g., the star/BD ratio $R$ or the median mass, is significantly affected by the choice of the isochrone used to estimated masses and the choice of the cluster parameters. Therefore, we caution against comparing IMF parameters derived using different assumptions.

2. If NGC 1333 is, in fact, closer to the Sun than IC348, as indicated by several independent studies, there is a significant difference in the mass distributions of these two clusters, in the sense that NGC 1333 harbors a larger fraction of very low mass stars and BDs.

3. The star/BD ratio is 1.9-2.4 in NGC 1333, consistent with previous estimates, and 2.9-4.0 in IC348, significantly lower than in previous estimates. The combined uncertainty in these values is approximately \pm 1 , but can be lowered with more accurate distance estimates and age estimates. If 
confirmed, these values would point to a larger fraction of BDs in NGC 1333.

4. These results ( 2 and 3 ) indicate that the relative number of very low mass objects in a star-forming regions may depend on the stellar density, in the sense that regions with higher density (such as NGC 1333) produce more very low mass objects. At this point, this conclusion is only based on two clusters and needs to be verified in other regions.

We thank Nickolas Moeckel, Gilles Chabrier, and Francesco Palla for helpful suggestions regarding topics discussed in this paper. A.S. acknowledges financial support through the grant 10/RFP/AST2780 from the Science Foundation Ireland. Additional support for this work came from grants to R.J. from the Natural Sciences and Engineering Research Council of Canada.

\section{REFERENCES}

Allard, F., Hauschildt, P. H., Alexander, D. R., Tamanai, A., \& Schweitzer, A. 2001, ApJ, 556, 357

Allard, F., Homeier, D., \& Freytag, B. 2011, in ASP Conf. Ser. 448, 16th Cambridge Workshop on Cool Stars, Stellar Systems, and the Sun, ed. C. Johns-Krull, M. K. Browning, \& A. A. West (San Francisco, CA: ASP), 91

Alves de Oliveira, C., Moraux, E., Bouvier, J., et al. 2013, A\&A, 549, A123

Andersen, M., Meyer, M. R., Greissl, J., \& Aversa, A. 2008, ApJL, 683, L183

Andersen, M., Meyer, M. R., Robberto, M., Bergeron, L. E., \& Reid, N. 2011, A\&A, 534, A10

Bally, J., Walawender, J., Johnstone, D., Kirk, H., \& Goodman, A. 2008, in Handbook of Star Forming Regions, Vol. I, ed. B. Reipurth (San Francisco: Astron. Society Pacific), 308

Baraffe, I., Chabrier, G., Allard, F., \& Hauschildt, P. H. 2002, A\&A, 382, 563

Bastian, N., Covey, K. R., \& Meyer, M. R. 2010, ARA\&A, 48, 339

Basu, S., \& Vorobyov, E. I. 2012, ApJ, 750, 30

Bate, M. R. 2012, MNRAS, 419, 3115

Belikov, A. N., Kharchenko, N. V., Piskunov, A. E., Schilbach, E., \& Scholz, R.-D. 2002, A\&A, 387, 117

Bonnell, I. A., Clark, P., \& Bate, M. R. 2008, MNRAS, 389, 1556

Bonnell, I. A., Larson, R. B., \& Zinnecker, H. 2007, in Protostars and Planets V, ed. B. Reipurth, D. Jewitt, \& K. Keil (Tucson, AZ: Univ. Arizona Press), 149

Cambrésy, L., Petropoulou, V., Kontizas, M., \& Kontizas, E. 2006, A\&A, 445,999

Cameron, E. 2011, PASA, 28, 128
Cardelli, J. A., Clayton, G. C., \& Mathis, J. S. 1989, ApJ, 345, 245

Cernis, K. 1990, Ap\&SS, 166, 315

Chabrier, G. 2003, PASP, 115, 763

Close, L. M., Lenzen, R., Guirado, J. C., et al. 2005, Natur, 433, 286

Da Rio, N., Robberto, M., Hillenbrand, L. A., Henning, T., \& Stassun, K. G. 2012, ApJ, 748, 14

Dawson, P., Scholz, A., Ray, T. P., et al. 2013, MNRAS, 429, 903

de Zeeuw, P. T., Hoogerwerf, R., de Bruijne, J. H. J., Brown, A. G. A., \& Blaauw, A. 1999, AJ, 117,354

Gieles, M., Moeckel, N., \& Clarke, C. J. 2012, MNRAS, 426, L11

Goodwin, S. P., \& Whitworth, A. 2007, A\&A, 466, 943

Gutermuth, R. A., Megeath, S. T., Myers, P. C., et al. 2009, ApJS, 184, 18

Gutermuth, R. A., Myers, P. C., Megeath, S. T., et al. 2008, ApJ, 674, 336

Haisch, K. E., Jr., Lada, E. A., \& Lada, C. J. 2001, ApJL, 553, L153

Helling, C., Ackerman, A., Allard, F., et al. 2008, MNRAS, 391, 1854

Hennebelle, P., \& Chabrier, G. 2009, ApJ, 702, 1428

Hirota, T., Honma, M., Imai, H., et al. 2011, PASJ, 63, 1

Irwin, J., Aigrain, S., Hodgkin, S., et al. 2007, MNRAS, 380, 541

Jørgensen, J. K., Harvey, P. M., Evans, N. J., II, et al. 2006, ApJ, 645, 1246

Jørgensen, J. K., Johnstone, D., Kirk, H., et al. 2008, ApJ, 683, 822

Kroupa, P. 2001, MNRAS, 322, 231

Lada, C. J., Alves, J., \& Lada, E. A. 1996, AJ, 111, 1964

Lombardi, M., Lada, C. J., \& Alves, J. 2010, A\&A, 512, A67

Luhman, K. L. 1999, ApJ, 525, 466

Luhman, K. L., \& Potter, D. 2006, ApJ, 638, 887

Luhman, K. L., Stauffer, J. R., Muench, A. A., et al. 2003, ApJ, 593, 1093

Moeckel, N., Holland, C., Clarke, C. J., \& Bonnell, I. A. 2012, MNRAS, 425,450

Muench, A. A., Lada, E. A., Lada, C. J., et al. 2003, AJ, 125, 2029

Padoan, P., \& Nordlund, A. 2002, ApJ, 576, 870

Prusti, T. 2011, in Stellar Clusters \& Associations: A RIA Workshop on Gaia, ed. E. J. Alfaro Navarro, A. T. Gallego Calvente, \& M. R. Zapatero Osorio (Grenada, Spain: European Space Agency), 11

Schlegel, D. J., Finkbeiner, D. P., \& Davis, M. 1998, ApJ, 500, 525

Scholz, A., Geers, V., Jayawardhana, R., et al. 2009, ApJ, 702, 805

Scholz, A., Muzic, K., Geers, V., et al. 2012, ApJ, 744, 6

Shen, S., Wadsley, J., Hayfield, T., \& Ellens, N. 2010, MNRAS, 401, 727

Stamatellos, D., \& Whitworth, A. P. 2009, MNRAS, 392, 413

Stassun, K. G., Kratter, K. M., Scholz, A., \& Dupuy, T. J. 2012, ApJ, 756, 47

Stassun, K. G., Mathieu, R. D., \& Valenti, J. A. 2007, ApJ, 664, 1154

Thies, I., Kroupa, P., Goodwin, S. P., Stamatellos, D., \& Whitworth, A. P. 2010, ApJ, 717, 577

Whitworth, A., Bate, M. R., Nordlund, Å., Reipurth, B., \& Zinnecker, H. 2007, in Protostars and Planets V, ed. B. Reipurth, D. Jewitt, \& K. Keil (Tucson, AZ: Univ. Arizona Press), 459

Wolk, S. J., Bourke, T. L., \& Vigil, M. 2008, in Handbook of Star Forming Regions, Vol. II, ed. B. Reipurth (San Francisco: Astron. Society Pacific), 124

Wuchterl, G., \& Tscharnuter, W. M. 2003, A\&A, 398, 1081 